\title{
Desenvolvimento de uma plataforma para auxílio em tratamento fisioterapico "finger flex"
}

\section{Development of a platform to aid in physiotherapy treatment "finger flex}

DOI: $10.46814 /$ lajdv3n2-005

Recebimento dos originais: 23/12/2020

Aceitação para publicação: 26/02/2021

\author{
Francisco Marcelino Almeida de Araújo \\ Mestrado completo, doutorando em Robótica \\ Instituto Federal do Piauí
}

Endereço: R. Álvaro Mendes, 94 - Centro (Sul), Teresina - PI, 64000-040

E-mail: franciscomarcelino@ifpi.edu.br

\section{Carla Maria de Carvalho Leite}

Doutora

Instituição de atuação atual: Universidade Federal do Piauí

Endereço: Campus Universitário Ministro Petrônio Portella - Ininga, Teresina - PI, 64049-550

E-mail: carlaanatomia@gmail.com

Francisco Vinicius Teles Rocha

Ensino médio completo, acadêmico em Medicina

Universidade Federal do Piauí

Endereço: Campus Universitário Ministro Petrônio Portella - Ininga, Teresina - PI, 64049-550

E-mail: fviniciustr@gmail.com

\author{
Amanda da Silva Souza \\ Especialista em Robótica Educacional \\ Universidade Estadual do Piauí \\ Endereço: Av. Nossa Sra. de Fátima, s/n - Nossa Sra. de Fátima, Parnaíba - PI, 64202-220 \\ E-mail: amandasouzaphb@gmail.com
}

\section{RESUMO}

O protótipo Finger Flex foi idealizado no intuito de ser uma nova ferramenta no acompanhamento da evolução de pacientes que precisem executar movimentos de repetição contínua, tudo isso em forma de uma interface interativa, que tem o objetivo de fazer com que os pacientes realizem os movimentos característicos do tratamento fisioterápico de uma maneira divertida, tornando-a mais agradável e menos monótona. O protótipo é constituído de uma luva com 5 sensores flexíveis acoplados a ela e de acordo com a movimentação realizada causará uma variação da tensão captada pela plataforma Arduino, o qual enviará as informações para uma interface construída usando a linguagem Processing, que com a plataforma Arduino são softwares livres. Posteriormente, os dados coletados serão enviados para um sistema que guarda todas as informações dos movimentos realizados durante a sessão.

Palavras Chaves: Arduino Lilypad, Processing, Sensor flexível, Fisioterapia, Tratamento fisioterápico. 


\section{ABSTRACT}

The prototype Finger Flex was designed in, to be a new tool for monitoring the progress of patients who need perform continuous repetition of movements, all in a game format that aims of make patients to they do characteristic movements of physical therapy in an entertaining way, making it more enjoyable and less monotonous. The prototype consists of a flexible glove with 5 sensors coupled her and according to the movement held to cause a variation of the voltage captured the Arduino platform, which sends the information to an interface built using the Processing language that with the Arduino platform are open source. Subsequently, the collected data will be sent to a system that stores all the information seen during the session.

Keywords: Arduino Lilypad, Processing IDE, Flex sensor, Physiotherapy, Physiotherapy treatment.

\section{INTRODUÇÃO}

Infecções, esforço repetitivo, quedas, acidentes de trânsito são algumas das causas de lesões que podem levar a diminuição da capacidade funcional da mão humana acarretando a Tenossinovite Estenosante, popularmente conhecida como dedo em gatilho, devido ao formato em que a mão afetada por essa enfermidade se encontra quando afetada por esta enfermidade.

Está é uma das doenças mais frequentes da mão e ocorre devido a um processo inflamatório crônico dos tendões flexores na região da base dos dedos que cursa com a diminuição da amplitude dos movimentos articulares dos dedos (BIASE, 2010).

Diante da gravidade desse mal, procedimentos de reabilitação são indispensáveis no seu tratamento. Um desses procedimentos, é a realização de movimentos de repetição contínua e ordenada dos dedos da mão afetada. De modo a promover o fortalecimento da musculatura dos tendões e articulações (NETO, 2010).

Em contrapartida, os profissionais desta área possuem ferramentas, porém, limitadas sendo menos precisas para controlar a medição da angulação. O goniômetro de dedo é um dos exemplos de ferramentas mais utilizadas para esses procedimentos, porem as informações podem diferir quando o processo de medição de um determinado paciente for realizado por mais de um profissional.

Pensando nesses recursos limitados, buscou-se uma solução através da robótica, na qual é proposto um protótipo em auxílio aos métodos hoje empregados. Ele é munido de sensores flexíveis, colocados um em cada dedo fazendo-se a leitura da angulação simultânea, onde essas leituras aparecerão na tela. Dessa forma, as sessões de fisioterapia deixarão de ser monótonas pois serão feitos através de um jogo, diminuindo o índice de evasão nas sessões, que se deve aos pacientes não encontrarem incentivo para o término do seu tratamento. 
Este trabalho tem como característica a utilização de hardware e software livres. Em pesquisas feitas encontrou-se trabalhos similares, porem eles utilizam videogames que eram feitas adaptações de jogos e controles para o uso em tratamentos de fisioterapia.

\section{TRABALHO PROPOSTO}

A hipótese trabalhada foi a de que o protótipo servisse como ferramenta que monitora a angulação de todos os dedos de forma simultânea, sendo uma alternativa aos métodos de hoje empregados na fisioterapia, tudo isso em formato de uma interface interativa, que tem o objetivo de fazer com que os pacientes realizem os movimentos característicos do tratamento fisioterápico para a para a Tenossinovite Estenosante.de uma maneira mais divertida e menos monótona. Dessa forma, além do cunho científico esse trabalho seria um incentivo para que os pacientes que necessitem fazer sessões de fisioterapia concluam o seu tratamento com o maior êxito possível.

Com esse intuito o trabalho constitui de uma plataforma em formato de jogo, no qual um paciente interagirá a medida que está fazendo as sessões de tratamento de reabilitação. A Plataforma é formada por uma luva que capta o movimento de todos os dedos, um jogo com variados níveis de dificuldades e um sistema que capta a movimentação da articulação dos dedos gerando dados, estes dados serão computados em gráficos. A imagem a seguir mostra a composição da plataforma citada anteriormente.

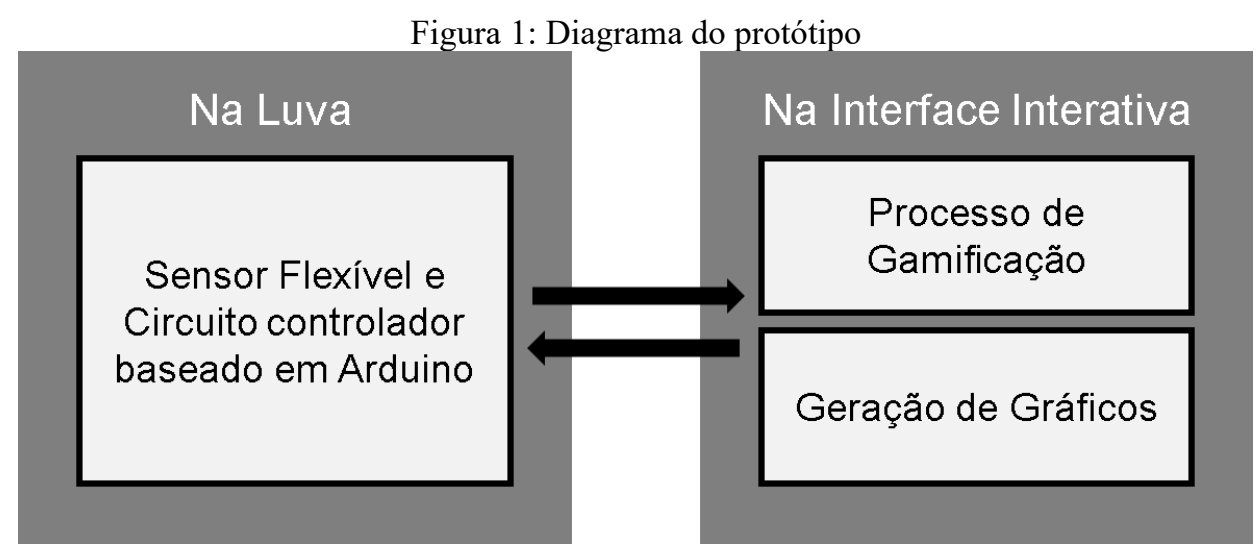

O funcionamento desse protótipo baseia-se na mudança da angulação dos sensores flexíveis, que resulta numa consequente variação de resistência deles. Através desse procedimento o microcontrolador Arduino Lilypad percebe uma variação de tenção e manda informações à interface do Processing IDE, que com a flexão dos dedos faz as bolas se moverem proporcionalmente (figura 2). Os dados colhidos dos movimentos dos dedos também são enviados para o sistema que por sua vez é 
responsável por gravar essas informações em um bando de dados para posterior consulta. O sistema também será responsável por gerar os gráficos de desempenho do paciente.

A plataforma possui três níveis (figura 3), sendo eles de dificuldade progressiva seguindo o seguinte padrão: No nível 1 o indivíduo deverá articular os dedos a um ângulo de $0^{\circ}$ a $30^{\circ}$, o segundo nível o indivíduo deverá articular os dedos a um ângulo de $0^{\circ}$ a $70^{\circ}$ e no terceiro nível o indivíduo deverá articular os dedos a um ângulo de $0^{\circ}$ a $120^{\circ}$.

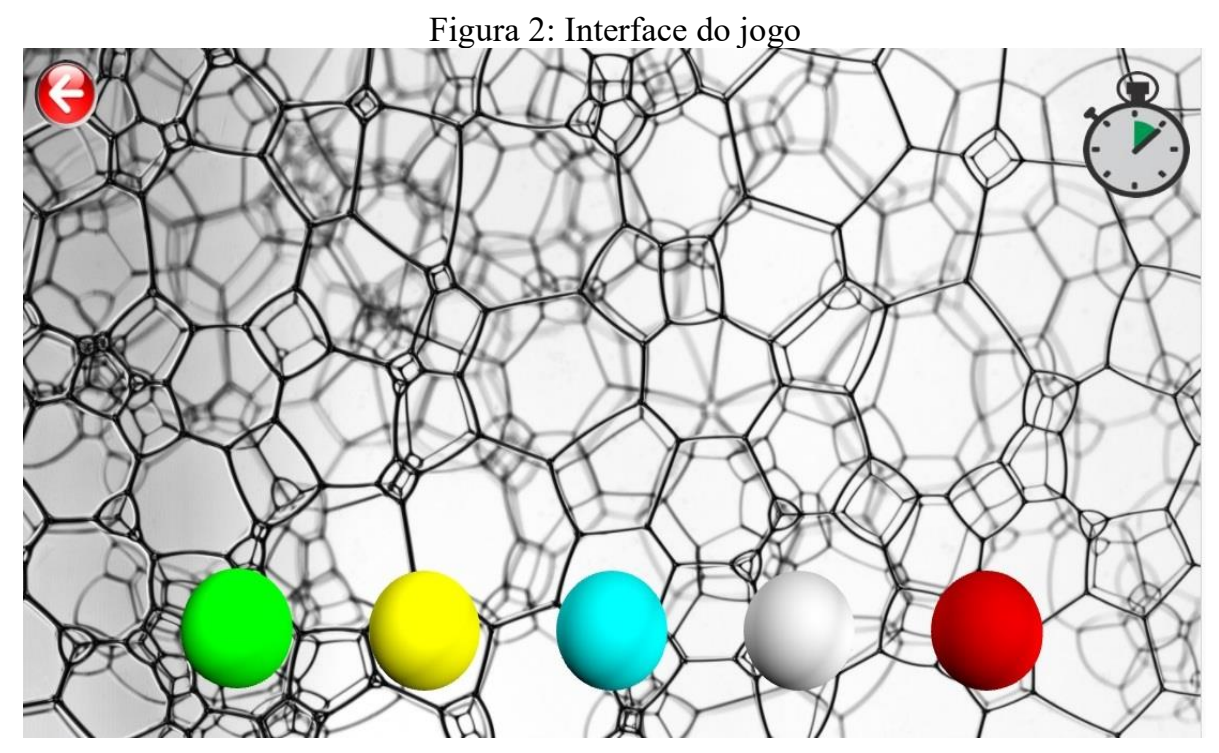

Figura 3: Interface de seleção de nível

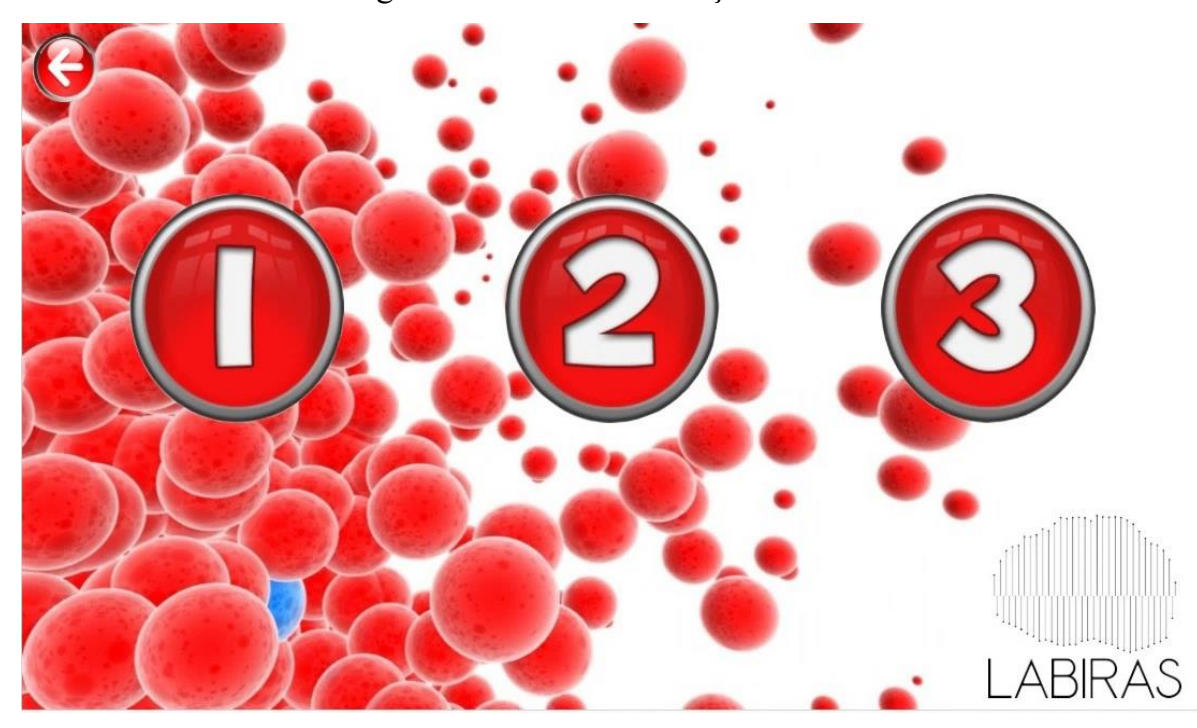

Cada sessão terá a duração de três minutos em todos os níveis, ao término deste tempo serão gerados os gráficos de rendimento do indivíduo para uma possível avaliação de um profissional da área, onde ele poderá diminuir ou aumentar o nível da sessão conforme for necessário. 


\section{MATERIAIS E MÉTODOS}

A plataforma foi desenvolvida utilizando o microcontrolador Arduino Lilypad e o sensor flex (Figura 3) para a construção da luva. A escolha deste microcontrolador deve-se pelas suas características, pois ele foi desenvolvido para ser trabalhado com vestuário, podendo ser lavado e costurado em peças de roupa, além de ser compacto e com isso suprindo com as necessidades do projeto.

O sensor flex tem por características medir a resistência aplicada a ele quando estiver curvado, tendo em vista, este sensor pode medir o quanto um dedo foi flexionado repassando os valores para o microcontrolador.

O protótipo foi submetido a alguns testes para a validação de suas funções. Primeiro foram feitos testes usando potenciômetros no lugar dos sensores flexíveis, pois assim como estes últimos são dispositivos que alteram sua resistência através de algum fator externo. Ao fazer estes testes tinha como objetivo verificar a funcionalidade do sistema criado.

Figura 4: Arduino Lilypad e Sensor Flexível

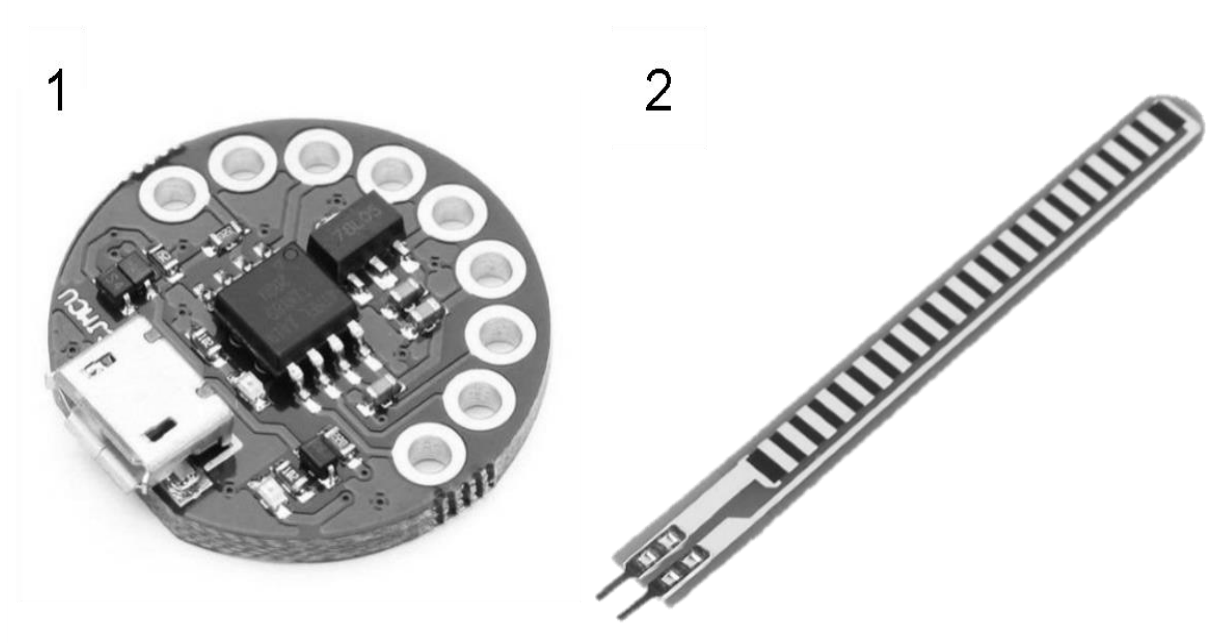

Legenda: 1: Arduino Lilypad; 2 Sensor Flexível

Em um segundo teste serão usados os sensores flexíveis fora da luva com o intuito de fazer sua calibração, pois eles devem ser usados em conjunto com outros resistores, construindo o esquema de um divisor de tensão (figura 5) para verificar a extensão do movimento da esfera quando se movimenta o dedo.

No terceiro teste os sensores serão acoplados a luva para a verificação e construção de gráficos de desempenho de um indivíduo através dos dados recebidos pelo microcontrolador, desta forma teremos um gráfico com duas linhas para cada dedo, como pode ser demonstrado n gráfico 1. 
Figura 5 - Diagrama do divisor de tensão

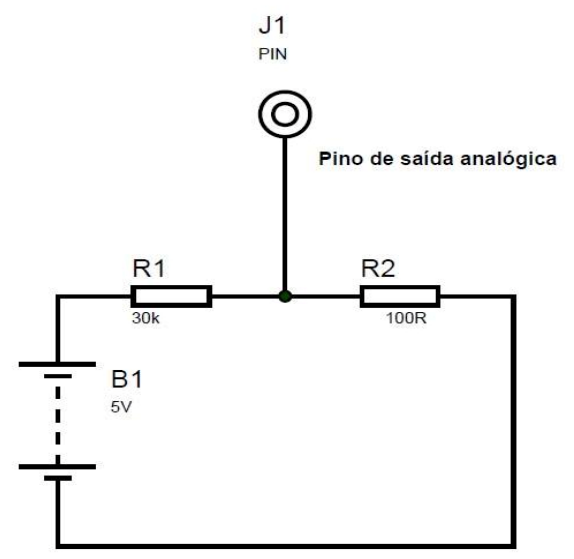

\section{RESULTADOS E DISCUSSÃO}

O funcionamento desse protótipo baseia-se na mudança da resistência elétrica dos sensores flexíveis. Os valores dessa resistência elétrica são convertidos em valores de tensão através dos divisores de tensão conectados ao Arduino Lilypad.

Através desse procedimento o microcontrolador Arduino Lilypad percebe uma variação de tensão em suas entradas analógicas e manda esses dados para uma interface interativa desenvolvida na linguagem Processing.

O que se esperou dos testes foi a correspondência entre a flexão das falanges e a movimentação das esferas na interface interativa, de modo que visualmente as bolas se movesse para cima proporcionalmente a flexão dos dedos (Figura 2).

Os resultados obtidos nos testes foram satisfatórios. Verificando a boa funcionalidade do sistema, o êxito na projeção dos divisores de tensão e calibração dos sensores na luva para cada usuário que venha a utilizar tal ferramenta possibilitando a construção de gráficos com os valores máximos e mínimos da angulação captada por cada sensor, onde ajudarão o profissional da área a ter um melhor acompanhamento da evolução do paciente.

Para cada usuário que venha a utilizar tal ferramenta é possível a captura dos dados das sessões e esses dados ficaram salvos em um banco de dados que posteriormente poderão ser requisitados para uma possível análise.

A plataforma teve um custo de produção acessível em consequência da utilização de recursos de software OpenSource, softwares gratuitos. Os custos de hardware podem ser vistos na Tabela 1. 
Tabela 1 - Custos do Produto

\begin{tabular}{|c|c|}
\hline Material & Custo em reais (cotação do dólar R\$ 4.44) \\
\hline Sensor Flex & $\mathrm{R} \$ 15,90$ \\
\hline Arduino Lilypad & $\mathrm{R} \$ 110,84$ \\
\hline
\end{tabular}

Figura 6- Gráfico de Desempenho do Dedo Indicador

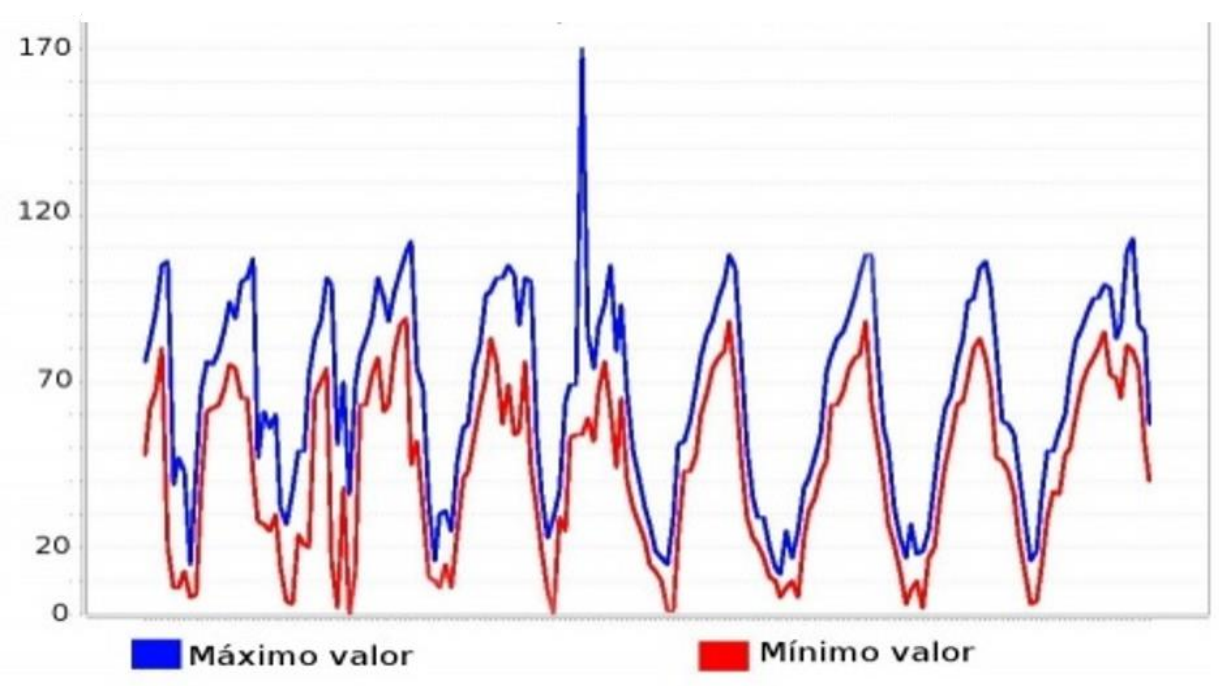

\section{CONCLUSÕES}

Segundo o que foi apresentado, a plataforma proporciona uma automação da fisioterapia tornando o tratamento mais estimulante. $\mathrm{O}$ ponto forte desse protótipo é que os pacientes poderão acompanhar de forma visual seu tratamento, pois todo ele será feito através de uma interface interativa.

O ponto negativo o protótipo é que diferentemente do goniômetro, não se pode saber com exatidão a angulação dos dedos. Sabendo-se somente a amplitude da extensão do movimento (Se o movimento foi curto, médio ou longo).

Este protótipo foi direcionado a mão, entretanto protótipos semelhantes poderão ser futuramente projetados para outras partes do corpo, de modo a ser feito um procedimento de sensoriamento semelhante.

\section{AGRADECIMENTOS}

Agradecemos ao LABIRAS (Laboratory of Intelligent Robotics, Automation and Systems) por incentivo e ceder o local onde ocorreram os estudos para o trabalho. 


\section{REFERÊNCIAS BIBLIOGRÁFICAS}

HELIO A. F. FONTES, Lesões Musculares In:. [s.n.], 2013. Disponível em: $<$ http://www.copacabanarunners.net/lesoes-musculares.html $>$, Acesso em: 07 de jun. De 2014

SCHMIDT MJ, ADAMS SL. Tendinopathy and bursitis. In: Marx JA, ed. Rosen"s Emergency Medicine: Concepts and Clinical Practice. 7a ed. Philadelphia, Pa: Mosby Elsevier;2009:cap 115.

NETO. J. M. Lesões musculares: diagnóstico, prevenção e tratamento. In:. [s.n.], 2010. Disponível em: $\quad<$ http://www.webrun.com.br/h/noticias/lesoes-musculares-diagnostico-prevencao-etratamento/11097>. Acesso em: 20 de jun. de 2014

ARDUINO. LilyPad Arduino USB. 2014. Disponível em: $<$ http://arduino.cc/en/Main/ArduinoBoardLilyPadUSB>. Acesso em: 07 de abr. de 2014

LEONARD A. Lesão Muscular: Conceitos e tratamentos. In:. [s.n.], 2013. Disponível em: $<$ http://www.adrianoleonardi.com.br/lesao-muscular-conceitos-e-tratamento/ >. Acesso em: 19 de jun. de 2014

PROCESSING. Processing. In:. [s.n.], 2014. Disponível em: <https://www.processing.org/>. Acesso em : 20 de jun. de 2014.

CASTRO, B. Estudantes criam jogo para ajudar pacientes. In:. [s.n.], 2011. Disponível em: $<$ http://g1.globo.com/jornal-hoje/noticia/2011/11/estudantes-criam-jogo-para-ajudar-pacientesrecuperar-movimentos.html>. Acesso em: 07 de jul. de 2014

PES-UNIFOR. Avanço da fisioterapia e o uso de novas tecnologias. In:. [s.n.], 2011. Disponível em: $<$ http://pefunifor.blogspot.com.br/v>. Acesso em: 07 de jul. de 2014

RODRIGUES, J. Video-game Wii põe diversão na fioterapia. In:. [s.n.], 2009. Disponível em: $<$ http://veja.abril.com.br/noticia/saude/videogame-wii-poe-diversao-fisioterapia $>$. Acesso em: 07 de jul. de 2014

Flexion. $\quad$ Sensorwiki. 15 nov. 2011, Disponivel em $<$ http://www.sensorwiki.org/doku.php/sensors/flexion>. Acesso em: 11 de jul. de 2014.

WATKINS A M; RIDDIE D L; PERSONIUS W J. Reliability of Goniometric Measurements and Visual Estimates of Knee Range of Motion Obtained in a Clinical Setting. Physical Therapy, Volume 71, Number 2, February, 1991 